\title{
Optimistic Fair Exchange of Digital Signatures
}

\author{
(Extended Abstract)
}

\author{
N. Asokan Victor Shoup Michael Waidner \\ IBM Zürich Research Laboratory, Säumerstr. 4, 8803 Rüschlikon, Switzerland \\ \{aso, sho, wmi\} 6 zurich.ibm.com
}

\begin{abstract}
We present a new protocol that allows two players to exchange digital signatures over the Internet in a fair way, so that either each player gets the other's signature, or neither player does. The obvious application is where the signatures represent items of value, for example, an electronic check or airline ticket. The protocol can also be adapted to exchange encrypted data. The protocol relies on a trusted third party, but is "optimistic," in that the third party is only needed in cases where one player attempts to cheat or simply crashes. A key feature of our protocol is that a player can always force a timely and fair termination, without the cooperation of the other player.
\end{abstract}

\section{Introduction}

As more business is conducted over the Internet, the fair exchange problem assumes increasing importance. For example, suppose player $A$ is willing to give an electronic check to player $B$ in exchange for an electronic airline ticket. The problem is this: how can $A$ and $B$ exchange these items so that either each player gets the other's item, or neither player does.

Both electronic checks and electronic airline tickets are implemented as digital signatures. Presumably, many other items to be exchanged over the Internet will be so implemented. Therefore, it seems fruitful to focus our attention on the fair exchange of digital signatures.

In this paper, we present a new protocol for the fair exchange of commonly used digital signatures, including RSA [21], DSS [16], Schnorr [22], Fiat-Shamir [12], GQ [15], and Ong-Schnorr [19] signatures, as well as the payment transcripts used in Brands' [7] off-line, anonymous cash scheme. The protocol can also be used to exchange data (such as stock quotes or an audio or visual stream) encrypted under a key derived from an RSA inverse or discrete logarithm. This scenario was suggested by Franklin and Reiter [13], where it was also suggested that the "quality" of the data be certified or guaranteed by an independent authority.

Our protocol uses a trusted third party, but only in a limited fashion: the third party is only needed in cases where one player attempts to cheat or simply crashes; therefore, in the vast majority of transactions, the third party will not need to be involved at all. Following [1], we call our protocol optimistic; in addition to [1], optimistic protocols for several variants of the fair exchange problem are discussed in $[8,18]$. 
Of course, one could use an on-line trusted third party $[10,11,14]$ in every transaction to act as a mediator, but the optimistic approach greatly reduces the load on the third party, which in turn reduces the cost and insecurity involved in replicating the service in order to maintain availability. It also makes it more feasible to distribute the third party, eliminating the single point of failure.

Our protocol also enjoys the following properties:

(1) To use it, one need not modify the signature scheme or message format at all. Thus, it will inter-operate with existing or proposed schemes for electronic checks, coins, tickets, receipts, etc., without any modification to these schemes.

(2) It works in an asynchronous communication model: there are no synchronized clocks, and one player cannot force the other to wait for any length of timea fair and timely termination can always be forced by contacting the third party.

(3) It is quite practical. A typical exchange requires only a few rounds of interaction, transmission of a few KBytes of data, and a couple thousand modular multiplications.

(4) The protocol can be proved secure (modulo standard intractability assumptions) in the random hash function model [5], where a hash function is replaced by a "black box" that outputs random bit strings.

(5) The two players may remain anonymous, if desired.

We stress the practical importance of property (1): it allows a general-purpose fair exchange service to be deployed without the cooperation of the institutions responsible for the items being exchanged (banks, airlines, etc.). Indeed, it seems quite unrealistic to expect these institutions to redesign their schemes and all of the relevant software to accommodate a fair exchange protocol if this has not already been designed for. Our protocol can accommodate any common signature scheme without modification. Previous optimistic protocols for fair exchange do not allow for this: these protocols either require that the item being exchanged have a special structure to facilitate the exchange protocol, or they partially sacrifice fairness, with one player ending up with just an affidavit from the third party that the other player owes him something. In our protocol, the two players get the real thing - not a substitute or affidavit.

Property (2) is perhaps even more important. Previous optimistic fair exchange protocols employ a "time-out" mechanism that either leaves one player "hanging" for an unacceptably long time (if the time-out is too long), or exposes the other player to an unacceptable risk of being cheated (if the time-out is too short). Not only can this be a great inconvenience, it can also lead to a real loss in the case of time-sensitive data like stock quotes. In our protocol, this cannot happen so long as the third party is available.

\subsection{Overview}

At a high level, our protocol works as follows. Suppose the two players hold signatures on agreed-upon messages. 
Each player first reduces a "promise" of a signature to "promise" of a particular homomorphic inverse (either a discrete logarithm or RSA inverse). A good reduction scheme should not make it any easier to compute the signature, while simultaneously guaranteeing that the promised signature can be recovered from the promised inverse. Good reduction schemes exist for all of the signature schemes mentioned above. Essentially the same notion of reduction was used in [13] (although their reduction scheme for DSS is actually insecure).

After this reduction phase, the players perform a fair exchange of homomorphic inverses. As mentioned above, this sub-protocol can be used in exchanging data encrypted under a key derived (perhaps via a hash function) from a homomorphic inverse. This idea is explored in further detail in [13].

To exchange homomorphic inverses, our protocol makes use of "verifiable" encryptions of these inverses under the third party's public key: such an encryption can be verified to contain the homomorphic inverse, without leaking any information that makes it easier to compute the inverse. For this, we adapt Bellare and Goldwasser's [4] key escrow technique.

Our protocol also employs a novel strategy to deal with the asynchrony problem discussed above, allowing the exchange to be "aborted" under certain conditions. This same strategy is also used in the companion paper [3] to design a protocol for contract signing that has the nice property that a cheating third party will eventually be caught.

The rest of the paper is organized as follows. In $\S 2$, we present a formal security model for the problem of exchanging digital signatures fairly. In $\S 3$, we show how to reduce the promise of a digital signature to the promise of a homomorphic inverse. In $\S 4$, we show how to verifiably encrypt a homomorphic inverse. Finally, in $\$ 5$, we present our optimistic protocol for the fair exchange of digital signatures.

A full length version of this paper is available [2].

\section{A Formal Security Model for Fair Signature Exchange}

We have two players $A$ and $B$, and a trusted third party $T$ that acts as a server: it receives a request from a client, updates its internal state, and sends a response back to the client. $T$ has a public key known to $A$ and $B$, and is always assumed to be honest and (eventually) available. We assume the client/server channel is private, and that server responses are authenticated, but do not assume that the client requests are authenticated. ${ }^{1}$

The two players agree upon the signatures they want to exchange, and then exchange messages back and forth. Between the time that a player receives a

1 This can be implemented by having the client encrypt its request together with a session key under the third party's public key; the server encrypts and authenticates its response using the given session key; this implementation will have the desired properties provided the third party's encryption scheme is secure against adaptive chosen ciphertext attack. 
message and generates its response, it may send requests to $T$, obtaining the corresponding responses within a finite, but unbounded, amount of time.

We define security in terms of farrness and completeness.

To define fairness, we let an adversary play the role of a corrupt player, and give it complete control over the network, arbitrarily interacting with $T$, and arbitrarily delaying $A$ 's requests to $T$. Intuitively, fairness means that it is infeasible for the adversary to get the honest player's signature, without the honest player getting the adversary's signature.

Completeness means that if neither player is corrupt, and no messages are lost, then the exchange is successful.

We now make the above notions a bit more precise.

Behavior of $T . T$ is a polynomial-time interactive Turing machine that follows the program prescribed for it by the protocol. $T$ acts as a server, repeatedly accepting a request, updating its internal state, and generating a response. For simplicity, we assume that each request is processed atomically. $T$ has a public $\mathrm{key} /$ private key pair $\left(P K_{T}, S K_{T}\right)$ that is generated by a key generation algorithm prescribed by the protocol.

Behavior of an honest player. An honest player is a polynomial-time interactive Turing machine that follows the program prescribed for it by the protocol. It interacts with its environment through a sequence of rounds: in one round it receives a message, updates its internal state, and generates a response.

Before generating a response, it may access $T$ (perhaps several times). To do this, the player must explicitly signal its intention to contact $T$, and then wait on an externally generated signal before proceeding.

The initial state of an honest player is determined by a set of inputs: $P K_{T}, P K, m, \sigma, P K^{\prime}, m^{\prime}$. Here, $\sigma$ is a signature on message $m$ under the public key $P K$ that the player intends to give in exchange for a signature on $\boldsymbol{m}^{\prime}$ under the public key $P K^{t}$.

After a bounded number of rounds, an honest player stops and writes on a private tape an output $\sigma^{\prime}$. The player also externally signals that it has terminated.

Definition of fairness. Fix a particular signature scheme $\Sigma$, and consider the following game. The components in the game are an adversary, called $B^{*}$, which is a polynomial-time interactive Turing machine, an honest player, called $A$, as well as $T, T$ 's key generation algorithm, and $\Sigma$ 's key generation algorithm.

\section{Game A}

A1 Run $\Sigma$ 's key generation algorithm, giving the secret key to a signing oracle $S$ and the public key $P K$ to $B^{*}$. Also generate $T$ 's public and private keys, giving $S K_{T}$ to $T$ and $P K_{T}$ to $B^{*}$.

A2 $B^{*}$ interacts arbitrarily with $T$ and $S$, obtaining signatures on adaptively chosen messages. 
A3 $B^{*}$ selects messages $m$ and $m^{\prime}$, and an arbitrary public key $P K^{\prime}$ for a signature scheme (possibly different from $\Sigma$ ). The message $m$ must be different from those given to $S$ in A2. Now, $S$ produces a signature $\sigma$ on $m$, and $A$ is initialized with inputs: $P K_{T}, P K, m, \sigma, P K^{\prime}, m^{\prime}$. The signature $\sigma$ is not seen by $B^{*}$.

A4 $B^{*}$ interacts with $T, S$ and $A$ in an arbitrary fashion, subject to the following restrictions:

(1) $B^{*}$ may not query $S$ with $m$.

(2) When $A$ signals its intention to contact $T, B^{*}$ must eventually signal $A$ to let it proceed, after which $B^{*}$ refrains from contacting $T$ until $A$ is finished (i.e., generates a response or a signal).

(3) Unless $A$ has signaled termination, $B^{*}$ must eventually supply another input message.

Eventually, $A$ terminates and outputs a string $\sigma^{\prime}$, and $B^{*}$ also terminates, and outputs a string $\bar{\sigma}$. We say that $B^{*}$ wins the game if $\sigma^{\prime}$ is not a valid signature for $m^{\prime}$ under $P K^{\prime}$, but $\bar{\sigma}$ is a valid signature for $m$ under $P K$. We define fairness to mean that $B^{*}$ cannot feasibly win this game.

Remarks. Restriction (2) captures our intuitive requirement that $A$ can always reach $T$, but may be arbitrarily delayed. Despite restriction (3), real world "time outs" can be modeled quite simply: in the real world, if $A$ is waiting for a message, then a low-level communication protocol will eventually time out and give $A$ the message "?"; in our formal model, $B^{*}$ just gives $A$ this message directly. Also, note that in our definition, the player in the exchange protocol is not necessarily the holder of the signing key.

Definition of completeness. We define another game similar to that above, in which the adversary gets access to two signing oracles, $S$ and $S^{\prime}$, and initializes two honest players $A$ and $B$, who interact directly with each other. The adversary in this case can interact with $T$, but cannot interfere with the interaction of $A$ and $B$, except insofar as the adversary still has the power to schedule both $A$ 's and $B$ 's interactions with $T$. We omit the details of this game, which are straightforward. The real-world situation that this game models is that where that all messages are delivered without being seen or modified by the adversary, and neither player "times out" waiting for a message.

We define completeness to mean that it is infeasible for the adversary in the above game to prevent $A$ and $B$ from successfully exchanging their signatures.

\section{Reducing Signatures to Homomorphic Inverses}

In this section, we show how to reduce a "promise" of a signature to the "promise" of a particular homomorphic inverse. In this extended abstract, we only deal with RSA, Schnorr and DSS signatures. In the full version of the paper, we deal with the others mentioned in the introduction.

As a simple, motivating example, consider the standard hash-and-invert RSA signature (which is provably secure in the random hash function model [5]). Here, 
the signature on a message is simply the eth root of the hash of the message, viewed as a number modulo a given composite number. In this case, reduction is trivial: the promised homomorphic inverse is simply this $e$ th root.

Reduction schemes for other signature schemes are not so trivial. So as to be able to uniformly treat several signature schemes, we start with a formal definition of our requirements.

\subsection{Definition of a secure reduction}

Let $\Sigma$ be a signature scheme. A reduction scheme for $\Sigma$ consists of three efficient algorithms, a reduction algorithm reduce, a verification algorithm verify, and a recovery algorithm recover, and also associates to every public key $P K$ an efficiently computable group homomorphism $\theta: G_{1} \rightarrow G_{2}$.

- reduce takes as input $P K, m, \sigma$, where $P K$ is a public key for $\Sigma, m$ is a message, and $\sigma$ is signature on $m$ under $P K$. The output consists of $d \in G_{2}$, $c \in\{0,1\}^{*}$, and $s \in \theta^{-1}(d)$. The string $c$ encodes additional information that is used by the verification and recovery algorithms. reduce may fail on some "bad signatures," as long as these occur rarely (we need this for DSS).

- verify takes as input $P K, m, d, c$, and either accepts or rejects.

- recover takes as input $P K, m, c$, and $s \in G_{1}$, and outputs a string $\bar{\sigma}$.

A secure reduction scheme should satisfy three properties:

Completeness. If reduce $(P K, m, \sigma)=(d, c, s)$, then verify $(P K, m, d, c)$ accepts.

Soundness. It is infeasible for an adversary to find $P K, m, d, c$ such that the verify $(P K, m, d, c)$ accepts, but recover $(P K, m, c, s)$ is not a valid signature on $m$ for all $s \in \theta^{-1}(d)$.

Secrecy. It is infeasible for an adversary to win the following game:

\section{Game B}

B1 Run $\Sigma$ 's key generation algorithm, giving the secret key to a signing oracle $S$ and the public key $P K$ to the adversary.

B2 The adversary makes arbitrary queries to $S$.

B3 The adversary generates a message $m$ different from those given to $S$ in B2. Now $S$ generates a signature $\sigma$ on $m$ under $P K$, and the adversary is given $d, c$, where $(d, c, s)=\operatorname{reduce}(P K, m, \sigma)$.

B4 The adversary continues to query $S$ on messages different from $m$.

The adversary wins the game if it can output a valid signature on $m$.

Remarks. Clearly, our definition of a secure reduction scheme implies that the underlying signature scheme is secure against adaptive chosen-message attacks. A stronger definition could be formulated wherein the reduction scheme is "just as secure" as the underlying signature scheme, however secure it happens to be. Unfortunately, not all of our proofs achieve this. It is also possibly to consider more general reduction schemes; for example, the reduction procedure could be interactive. We do not consider these here. 


\subsection{Schnorr Signatures}

In the Schnorr signature scheme, to generate a public key, one selects primes $p$ and $q$ such that $q \mid p-1$, and a generator $g$ for the subgroup of $\mathbf{Z}_{p}^{*}$ of order $q$. One then chooses $x \in \mathbf{Z}_{q}$ at random and computes $h=g^{x}$. The public key consists of $p, q, g, h$, and the private key is $x$. To sign a message $m$, the signer chooses $r \in \mathbf{Z}_{q}$ at random, and computes $z=c x+r$, where $c=H\left(g^{r}, m\right) \in \mathbf{Z}_{q}$ and $H$ is a hash function. The signature is $(c, z)$. To verify a signature, one checks that $c=H\left(g^{z} h^{-c}, m\right)$.

The Schnorr signature scheme is provably secure in the random hash function model (if the discrete logarithm problem is hard).

The following reduction from Schnorr signatures to discrete logarithms was observed in [13], in the context of verifiable signature sharing. The reduction algorithm takes as input a signature $(c, z)$ on a message $m$, and outputs $u=g^{z}, c$, where the promised inverse is $z=\log _{g} u$.

The verification algorithm checks that $c=h\left(u h^{-c}, m\right)$. Given $z$, the recovery algorithm outputs $(c, z)$.

Completeness and soundness are clear. Secrecy follows from the fact that one can simulate the output of the reduction algorithm without a signature: the simulator chooses $r \in \mathbf{Z}_{q}$ at random, computes $w=g^{r}$ and $c=H(w, m)$, and sets $u=w h^{c}$.

\subsection{DSS Signatures}

Key generation for DSS is identical to that for the Schnorr scheme. The standard prescribes that $q$ has a length of 160 bits. To sign a message $m$, the signer chooses $k \in \mathbf{Z}_{q}^{*}$ at random, and computes $r=\left(g^{k}\right) \bmod q$ and $s=k^{-1}(H(m)+x r)$, where $H$ is a hash function with outputs in $\mathbf{Z}_{q}$. The signature is $(r, s)$. A signature is verified by checking that $r=\left(g^{u_{1}} h^{u_{2}}\right) \bmod q$, where $u_{1}=H(m) s^{-1}$, and $u_{2}=r s^{-1}$. Note that signatures with $s=0$ are invalid, but these effectively never arise. For our reduction scheme, we must also rule out $r=0$, which also effectively never arises.

We now give a reduction scheme reducing a promise of a DSS signature to a promise of discrete logarithm.

The reduction algorithm works as follows. We have a signature $(r, s)$ on a message $m$. Let $u_{1}, u_{2}$ be defined as above, and define $c=r^{-1} H(m)$. We output

$$
\alpha=g^{u_{1}}, \beta=h^{u_{2}}, \alpha^{\prime}=g^{v}, \beta^{\prime}=h^{v}, z=v+e u_{1},
$$

where $v \in \mathbf{Z}_{q}$ is chosen at random, $e=H^{\prime}\left(P K, \alpha, \beta, \alpha^{\prime}, \beta^{\prime}, c\right)$, and $H^{\prime}$ is a hash function.

The promised inverse is $u_{2}=\log _{h} \beta$.

The verification algorithm runs as follows. We first check that $\alpha^{q}=\beta^{q}=1$ and $\beta \neq 1$; we then compute $r=(\alpha \beta) \bmod q$, and check that $r \neq 0$, Finally, we compute $c=r^{-1} H(m), e=H^{\prime}\left(\alpha, \beta, \alpha^{\prime}, \beta^{\prime}, c\right)$, and check that $g^{z}=\alpha^{\prime} \alpha^{e}$ and $h^{z}=\beta^{\prime} \beta^{c e}$. 
What is happening here is that we are giving $\alpha$ and $\beta$, and a non-interactive proof that $\log _{g} \alpha=c \log _{h} \beta$ (see [9]).

The recovery procedure takes $u_{2}=\log _{h} \beta$, computes $r=(\alpha \beta) \bmod q$ and $s=r / u_{2}$, and outputs $(r, s)$.

To prove secrecy, we have to make a strong security assumption about DSS: given signatures on several messages, not only is it difficult for an adversary to compute a signature on a new message, it is difficult to compute a different signature on any of the given messages.

One can heuristically justify this assumption using the "generic algorithm" model in [23], assuming also that $H$ is collision free. Proving that DSS satisfies this stronger form of security in this model is relatively straightforward; moreover, this is the only model that we know of in which it is possible to prove that DSS is secure to begin with.

Lemma 1. Under the strong security assumption for DSS, above reduction scheme is secure in the random hash function model for $H^{\prime}$.

The proof is in Appendix A.

Remark. It has been brought to our attention that there are simpler reduction schemes for DSS; however, our reduction is still perhaps of some interest, as the promised discrete logarithm is relative to a fixed base, whereas in the simpler schemes it is relative to a random base; using a fixed base can lead to greater efficiency and also facilitates the use of certified encryptions discussed at the end of $\S 5$ and in more detail in the full-length version of this paper.

\section{Verifiable Encryption of Homomorphic Inverses}

Suppose we a have a surjective group homomorphism $\theta: G_{1} \rightarrow G_{2}$. We have a publicly known group element $d \in G_{2}$ and a secret $s \in \theta^{-1}(d)$. We want to encrypt $s$ under the public key of a third party in such a way that it can be publicly verified that when decrypted, an inverse of $d$ is obtained. However, we want to ensure that this verification procedure itself does not reveal any information that helps invert $d$. We also immutably bind to the encryption an label $x \in\{0,1\}^{*}$, which will be used by the third party to determine if this decryption is authorized.

More formally, a verifiable encryption scheme consists of a key generation algorithm, a prover $P$, a verifier $V$, a decryption algorithm $D$, and a recovery algorithm $R$. $P$ and $V$ have as a common inputs $d \in G_{2}$ and $x \in\{0,1\}^{*}$, along with the public encryption key. $P$ also has a private input $s \in \theta^{-1}(d)$. At the end of the protocol, $V$ either accepts and outputs a string $\alpha$, or rejects. The string $\alpha$ is a ciphertext that can be given to $D$, along with the label $x$; the output from $D$ can be given to $R$ to obtain $s$.

A secure verifiable encryption scheme satisfies the following properties:

Completeness. If both $P$ and $V$ are honest, then for all $s, d$, and $x$, with $\theta(s)=d, V$ accepts. 
Soundness. For all $d$ and $x$, and for arbitrary $P^{*}$, if $V$ accepts and outputs $\alpha$, then with overwhelming probability, $\theta(R(D(\alpha, x)))=d$.

Zero Knowledge. Consider the following game played against an adversary:

\section{Game C}

C1 The key generation algorithm is run, the private key is given to $D$ and the public key is given to the adversary.

C2 The adversary makes arbitrary queries $D\left(\alpha^{\prime}, x^{\prime}\right)$.

C3 The adversary generates $s, d, x$ with $\theta(s)=d$, and gives $P$ the input $s, d, x$, along with the public encryption key.

C4 The adversary makes arbitrary queries to $D$ and $P$, but after its first query to $P$, it may not query $D$ with label $x$.

A simulator is a machine that plays the roles of the key generation algorithm, the decryption oracle $D$, and the prover $P$, but is only given $d$ and $x$-and not $s$. In the random hash function model, the simulator also responds to the random function queries. Zero knowledge means there exists a simulator such that the adversary cannot feasibly distinguish between the real game and the simulated game.

Note that in the definition of zero knowledge, the power of the simulator is quite limited: it must respond to queries on-line, and is not allowed to do anything like "rewind" the adversary.

We now give our verifiable encryption scheme, which is already optimized somewhat to reduce the amount of data transmitted.

First, we assume that we have a public-key encryption function $E$ secure against adaptive chosen ciphertext attack [20]. More precisely, the public key defines a function $E(t, y)$; to encrypt a string $y$, one chooses a random string $t$, of length, say, $k$, and computes $E(t, y)$.

Second, we assume we have hash functions $H_{1}, H_{2}$, and $H_{3} . H_{1}$ takes a string $r$, of length, say $l$, and outputs a pair $\left(t, s^{\prime}\right) \in\{0,1\}^{k} \times G_{1} . H_{2}$ is a hash function that maps a pair $\left(\alpha, d^{\prime}\right) \in \operatorname{Range}(E) \times G_{2}$ to a short string. $H_{3}$ just hashes bit strings to shorter bit strings (but long enough to resist collisions). Also, a security parameter $N$ is defined.

The protocol is a simple "cut and choose" scheme (but with exponential-not linear-security), and runs as follows.

\section{Protocol D}

The following steps are executed $N$ times in parallel.

D1 $P$ chooses $r \in\{0,1\}^{l}$ at random, computes $\left(t, s^{\prime}\right)=H_{1}(r)$, and sends $h=$ $H_{2}\left(E\left(t,\left(s^{\prime}, H_{3}(x)\right)\right), \theta\left(s^{\prime}\right)\right)$ to $V$.

D2 $V$ chooses $b \in\{0,1\}$ at random, and sends $b$ to $P$.

D3 If $b=0, P$ sends $r$ to $V$. If $b=1, P$ sends $\alpha=E\left(t,\left(s^{\prime}, H_{3}(x)\right)\right)$ and $s^{\prime \prime}=s^{\prime}+s$ to $V$. 
D4 If $b=0, V$ computes $\left(t, s^{\prime}\right)=H_{1}(r)$ and checks that $h=$ $H_{2}\left(E\left(t,\left(s^{\prime}, H_{3}(x)\right)\right), \theta\left(s^{\prime}\right)\right)$. If $b=1, V$ checks that $h=H_{2}\left(\alpha, \theta\left(s^{\prime \prime}\right)-d\right)$. If these checks fail, $V$ rejects.

$V$ rejects if it rejects in any of the $N$ rounds. Otherwise, it accepts and outputs the set of all of the ciphertexts $\alpha$ in those rounds with $b=1$. Actually, to ensure completeness, $V$ should pick a random string of $N$ bits, not all zero.

Decryption is straightforward: when $D$ is given an label $x$ and a set of ciphertexts $\{\alpha\}$, each ciphertext is decrypted, and the first component of the cleartext is returned if the second component of the cleartext matches $H_{3}(x)$. The recovery algorithm takes the given values $s^{\prime}$, and computes $s=s^{\prime \prime}-s^{\prime}$ for the corresponding $s^{\prime \prime}$ in protocol $\mathrm{D}$, and outputs $s$ if $\theta(s)=d$ for one of these values.

Lemma 2. In the random hash function model for $H_{1}, H_{2}$, and $H_{3}$ the above scheme is a secure verifiable encryption scheme.

The proof is in Appendix B.

\subsection{An Example Implementation: Discrete Logarithms}

Assume that $H_{2}$ and $H_{3}$ have 160-bits of output, and that the input length $l$ of $H_{1}$ is 160 bits. Note that the probability that an honest verifier is "cheated" in any one interaction is roughly $2^{-N}$; therefore, $N=40$ should be sufficient for most applications. One could make the protocol non-interactive using standard techniques involving hash functions, but then much larger values of $N$, say $N=$ 80 , would be required to avoid off-line attacks.

For our encryption function, we take the OAE encryption function of Bellare and Rogaway [6], based on the RSA problem. Assume a composite modulus of 1024 bits and an encryption exponent of 3 . OAE is secure against chosen ciphertext attacks in the random hash function model (although the proofs in [6] have to be adapted slightly to prove this).

Apropos DSS, for the discrete logarithm problem, assume the group $G_{2}$ is the subgroup of order $q$ in $\mathrm{Z}_{p}^{*}$, where $p$ is a 1024-bit prime, and $q$ is a 160-bit prime. Let $g$ be the given generator for $G_{2}$. In our notation, the group $G_{1}$ is the additive group $\mathbf{Z}_{q}$, and $\theta$ sends $a \in \mathbf{Z}_{q}$ to $g^{a}$.

The expected amount of data transmitted is about 4 KBytes.

Both $P$ and $V$ perform 40160 -bit exponentiations in $\mathbf{Z}_{p}^{*}$, all to the base $g$, plus the multiplications for OAE. Using techniques of Lim and Lee [17], each party can do this using under 2000 modular multiplications. This bound already includes the precomputation time for the base $g$. If $g$ is actually fixed for one of the parties, this bound can be reduced to about 1000 modular multiplications.

\subsection{An Example Implementation: RSA Inverses}

Assume the same encryption function as above.

For the RSA inverse problem, assume a 1024-bit composite modulus $M$ and encryption exponent $e$ with $(\phi(M), e)=1$. In our notation, $G_{1}=G_{2}=\mathbf{Z}_{M}^{*}$, and 
$\theta(a)=a^{e}$. Typically, $e=3$ or $e=2^{16}+1$. One could also take $e=2$, in which case $G_{2}=\left(\mathbf{Z}_{m}^{*}\right)^{2}$.

The expected amount of data transmitted is about 7 KBytes.

For $e=3$, each party needs no more than 160 modular multiplications, and for $e=2^{16}+1$, this number is under 800 .

\section{The Fair Exchange Protocol}

Now that we have all the necessary tools, we can easily describe our fair exchange protocol.

Suppose $A$ holds a signature $\sigma_{A}$ on message $m_{A}$ under public key $P K_{A}$, and $B$ holds a signature $\sigma_{B}$ on message $m_{B}$ under public key $P K_{B}$

We make use of our scheme for reducing signatures to homomorphic inverses. Let $\theta_{A}$ be the relevant homomorphism for $A$ 's signature, and $\theta_{B}$ be that for $B$ 's signature. We write $\operatorname{desc}\left(\theta_{B}\right)$ for a string that describes the relevant groups and an algorithm for computing the homomorphism using some standard encoding.

We also make use of our scheme for verifiable encryption of homomorphic inverses under $T$ 's public key. Recall that when $T$ decrypts such a verified encryption, it always does this subject to a label bound to the encryption at the time the encryption was created.

We also make use of a one-way function $f$.

The third party $T$ maintains a set $S$ of tuples, whose structure is described below. We describe the protocol assuming $A$ makes the first move.

\section{Protocol E}

E1 $A$ computes reduce $\left(P K_{A}, m_{A}, \sigma_{A}\right)=\left(d_{A}, c_{A}, s_{A}\right)$, and sends $d_{A}, c_{A}$ to $B$.

E2 $B$ checks that verify $\left(P K_{A}, m_{A}, d_{A}, c_{A}\right)$ accepts; if not, $B$ halts. Otherwise, $B$ computes reduce $\left(P K_{B}, m_{B}, \sigma_{B}\right)=\left(d_{B}, c_{B}, s_{B}\right)$, and sends $d_{B}, c_{B}$ to $A$.

E3 $A$ checks that verify $\left(P K_{B}, m_{B}, d_{B}, c_{B}\right)$ accepts; if not, $A$ halts. Otherwise, $A$ chooses $r \in \operatorname{Domain}(f)$ at random and computes $v=f(r)$. A and $B$ then engage in the verifiable encryption protocol, with $A$ as prover and $B$ as verifier, so that $A$ gives to be $B$ a verified encryption $\alpha$ of $s_{A}$ with label $\left(v, d_{B}, \operatorname{desc}\left(\theta_{B}\right)\right)$.

E4 If $B$ rejects the proof in E3, then $B$ halts. Otherwise, $B$ and $A$ engage in the verifiable encryption protocol, with $B$ as prover and $A$ as verifier, so that $B$ gives to $A$ a verified encryption $\beta$ of $s_{B}$ with label $v$.

E5 If $A$ rejects the proof in E4, then $A$ invokes sub-protocol abort. Otherwise, $A$ sends $s_{A}$ to $B$.

E6 $B$ checks that $\theta_{A}\left(s_{A}\right)=d_{A}$. If not, $B$ invokes sub-protocol B-resolve. Otherwise, $B$ sends $s_{B}$ to $A$, outputs recover $\left(P K_{A}, m_{A}, c_{A}, s_{A}\right)$, and halts.

E7 $A$ checks that $\theta_{B}\left(s_{B}\right)=d_{B}$. If not, $A$ invokes sub-protocol A-resolve. Otherwise, $A$ outputs recover $\left(P K_{B}, m_{B}, c_{B}, s_{B}\right)$, and halts. 


\section{Sub-protocol abort}

$A$ sends $r, d_{B}, \operatorname{desc}\left(\theta_{B}\right)$ to $T$, who does the following:

if (deposit, $\left.f(r), d_{B}, s_{B}, \operatorname{desc}\left(\theta_{B}\right)\right) \in S$ then send $s_{B}$ to $A$, from which $A$ recovers $\sigma_{B}$ else if (no-abort, $f(r)) \in S$ then send $A$ the message "abort not allowed" else

add (abort, $f(r)$ ) to $S$ send $A$ the message "exchange aborted"

\section{Sub-protocol B-resolve}

$B$ sends $\alpha, v, s_{B}, \operatorname{desc}\left(\theta_{B}\right)$ to $T$, who does the following:

if (abort, $v$ ) $\in S$ then

send $B$ the message "exchange aborted" else

add (deposit, $\left.v, \theta_{B}\left(d_{B}\right), s_{B}, \operatorname{desc}\left(\theta_{B}\right)\right)$ to $S$;

decrypt $\alpha$ subject to the label $\left(v, \theta_{B}\left(s_{B}\right), \operatorname{desc}\left(\theta_{B}\right)\right)$, and send result to $B$, from which $B$ recovers $\sigma_{A}$.

\section{Sub-protocol A-resolve}

$A$ sends A-resolve, $\beta, r$ to $T$, who does the following:

if (abort, $f(r)) \in S$

send $A$ the message "exchange aborted" else

add (no-abort, $f(r)$ ) to $S$;

decrypt $\beta$ subject to the label $f(r)$,

and send result to $A$, from which $A$ recovers $\sigma_{B}$

Our main result is the following.

Theorem 3. Assuming that $f$ is one-way, that the underlying reduction and verifiable encryption schemes are secure (in the random hash function model), then the above fair exchange protocol is secure (in the random hash function model).

The proof is in Appendix C. The intuition for the proof of fairness is that at no point does the adversary obtain any useful information about the honest player's signature before it becomes essentially inevitable that the honest player will obtain the adversary's signature. 
Remarks. (1) Using standard techniques, $s_{A}$ and $s_{B}$ can be blinded, so that if both $A$ and $B$ are honest, $T$ obtains no useful information. (2) In principle, when a tuple is added to $S$, it must stay there forever; in practice, an "aging" mechanism can be introduced, allowing old tuples to be eventually flushed. (3) The technique of using verifiable encryptions could be replaced, under some circumstances, by a more efficient technique of certified encryptions. With this technique, a player using a fixed signing key could obtain a number of certified encryptions from a third party off-line, before the player knows what messages will be signed. Details are in the full version of the paper.

\section{Appendix A: Proof of Lemma 1}

Completeness. Clear.

Soundness. Let $u_{2}$ satisfying $h^{u_{2}}=\beta$ be given. Set $r=(\alpha \beta) \bmod q, s=r u_{2}^{-1}$, $u_{1}=H(m) s^{-1}$, and $c=r^{-1} H(m)$. The verification procedure verifies a noninteractive proof that $\log _{g} \alpha=c \log _{h} \beta$. This proof is sound assuming $H^{\prime}$ is a random function, so assume this identity holds. This, together with the identity $u_{1}=c u_{2}$, implies that $g^{u_{1}}=\alpha$, proving that $(r, s)$ is a valid signature.

Secrecy. Suppose that an adversary can win Game B, obtaining a signature on a message $m$ chosen in B3. Under the strong security assumption for DSS, this signature must be the same as that generated in B3, which implies the adversary can compute $u_{2}=\log _{h} \beta$.

We show how to use this adversary to compute discrete logarithms efficiently, assuming $H^{\prime}$ is a random function. Let $\omega$ be a random element of order $q$ in $\mathbf{Z}_{p}^{*}$ whose discrete logarithm to the base $g$ we wish to compute. We choose $x \in \mathbf{Z}_{q}$ at random, and compute $\boldsymbol{h}=\boldsymbol{g}^{x}$, and use $\boldsymbol{h}$ as a public key for the signature scheme. Since we know $x$, we can generate signatures for the adversary as necessary in B2 and B4. Now in B3, we are given a message $m$ by the adversary. We do not sign this message; instead, we compute $r=\omega \bmod q$, and set $c=r^{-1} H(m)$. Then we compute $\beta=\omega^{x /(x+c)}$ and $\alpha=\omega / \beta$. It is easily verified that $\alpha$ and $\beta$ have precisely the same distribution as in the actual reduction algorithm, so this part of the simulation is perfect. Also, since $H^{\prime}$ is a random function, we can easily simulate the proof that $\log _{g} \alpha=c \log _{h} \beta$, since the corresponding interactive proof is zero knowledge against an honest verifier. Thus, we have perfectly simulated the output of the reduction algorithm. Now if the adversary computes $u_{2}=\log _{h} \beta$, then we obtain $\log _{g} \omega=(x+c) u_{2}$.

\section{Appendix B: Proof of Lemma 2}

Completeness. Clear.

Soundness. This is a standard argument, relying only on the presumption that it is impossible to find collisions in $\mathrm{H}_{2}$. 
Zero Knowledge. We now describe the simulator $P^{\prime}$ that responds to the queries made to $P$ and the random hash functions. At any stage in Game $C$, responses to the hash function queries are simply made in a random, but consistent, fashion. In stage $\mathrm{C} 3$, the simulator receives inputs $d, x$. In stage $\mathrm{C} 4$, the simulator responds to queries made to $P$ as follows.

In step D1, $P^{\prime}$ just outputs $N$ random strings, representing $N$ outputs of $H_{2}$, whose inputs are yet to be determined. In step D3, $P^{\prime}$ does the following in each of the $N$ rounds, depending on the value of $b$ given in D2.

If $b=0, P^{\prime}$ picks $r \in\{0,1\}^{l}$ at random, and sets $\left(t, s^{\prime}\right)=H_{1}(r)$. Then it sets $\alpha=E\left(t,\left(s^{\prime}, H_{3}(x)\right)\right)$. Now $P^{\prime}$ has to "backpatch" $H_{2}$, making the output of $H_{2}$ at the point $\left(\alpha, \theta\left(s^{\prime}\right)\right)$ equal to the corresponding string output in step D1. It is easily verified that this can almost certainly be done consistently (and if not, we simply quit).

If $b=1, P^{\prime}$ chooses $s^{\prime \prime} \in G_{1}$ at random, and creates a "dummy" ciphertext $\alpha$ under $E$ of $\left(0, H_{3}(x)\right)$. Again, we have to backpatch $H_{2}$, this time at the point $\left(\alpha, \theta\left(s^{\prime \prime}\right)-d\right)$, and again it is easy to see that this backpatching can almost certainly be done consistently.

That completes the description of the simulator. We next claim that if the adversary can distinguish a simulated Game $C$ from a real Game $C$, we can use this adversary to break the underlying encryption scheme. To see this, note that in the simulated game, if the adversary ever presents one of the dummy ciphertexts for decryption in stage $\mathrm{C} 4$, with label $x^{\prime} \neq x$, then we do not have to give the underlying decryption algorithm for $E$ the ciphertext, since the decryption algorithm $D$ would almost certainly refuse to give the adversary the cleartext anyway. Then, using a standard argument, the adversary could be used to distinguish an encryption of $\left(0, H_{3}(x)\right)$ from an encryption of $\left(s^{\prime}, H_{3}(x)\right)$, thus breaking the underlying encryption scheme.

\section{Appendix C: Proof of Theorem 3}

Completeness is clear. To prove fairness, we start with an adversary that can win Game A. Consider first the case where $A$ is the honest player, and call the adversary $B^{*}$.

We begin by defining a truncated version of Game $A$, call it Game $A^{\prime}$, defined by the following two early-stopping rules:

(1) Suppose that E4 has just completed with $A$ accepting the proof in verifiable encryption protocol. Then we stop the game at this point, and say that $B^{*}$ loses.

(2) Suppose that $A$ has begun to engage in the encryption protocol in E3 with a given label, and $A$ has not executed the abort sub-protocol. If these conditions hold, and $B^{*}$ sends a request to $T$ for B-resolve with arguments that yield a matching label, we stop the game just before $T$ responds, and say $B^{*}$ loses.

We claim that if $B^{*}$ can win Game $A$, then he can also win Game $A^{\prime}$. To see why, consider stopping rule (1). Once $A$ accepts the verifiable encryption, 
it is effectively inevitable that $A$ would obtain $s_{B}$, and hence $\sigma_{B}$, in Game A: either directly in step E6, or via sub-protocol A-resolve. In the latter case, since $f$ is one-way, the adversary cannot block the A-resolve by having an abort tuple placed in $S$ via sub-protocol abort.

Now consider stopping rule (2). Since $A$ has not aborted the transaction (and only $A$ can do this as $f$ is one-way), then performing the B-resolve in Game A would result in a deposit tuple being placed on $S$ that contains an inverse of $s_{B}$. Then $A$ will eventually get $s_{B}$, and hence $\sigma_{B}$, either from $B^{*}$ directly in step E6, or from $T$ via abort or A-resolve, which again $B^{*}$ cannot prevent since it cannot invert $v$.

So assume $B^{*}$ can win Game $\mathbf{A}^{\prime}$. We now make another transformation: we replace the $A$ 's prover in the verifiable encryption protocol in E3 (and any random hash functions) with the corresponding zero-knowledge simulator. Because of the way we truncated Game A, we are guaranteed that $T$ 's decryption function will never be called with a matching label at any time after the prover starts.

Call this Game $A^{\prime \prime}$. By the zero-knowledge property, it follows that $B^{*}$ can also win Game $A^{\prime \prime}$. Moreover, when we play Game $A^{\prime \prime}$ against the adversary, we do not need the $s_{A}$ output from the reduction algorithm to run the prover simulation, and it is not in the adversary's view, so we can drop it altogether. The result is an adversary that breaks the supposed secrecy of $A$ 's reduction scheme.

That concludes the proof for the case where $A$ is the honest player. Now assume $B$ is the honest player, and call the adversary $A^{*}$. We use a very similar argument. We first define a truncated Game $A^{\prime}$ via three early-stopping rules:

(1) If $A^{*}$ sends $B$ a valid inverse in E5, we stop, and say that $A^{*}$ loses.

(2) If $B$ has completed step E3, accepting the proof in the verifiable encryption, and $A^{*}$ has not performed a successful abort with $v$, and then $A^{*}$ performs an A-resolve with $v$, then we stop the game just before $T$ responds, and say that $A^{*}$ loses.

(3) If $B$ has just accepted the proof E3, but $A^{*}$ has already performed a successful A-resolve with $v$, then we stop, and say that $A^{*}$ loses.

As before, we show that if $A^{*}$ can win Game $\mathrm{A}$, it can also win Game $\mathrm{A}^{\prime}$. Clearly, if we stop on rule (1), then in Game A, $B$ would recover $\sigma_{A}$, and so $A^{*}$ would lose. Also, if we stop on rules (2) or (3), then in Game A, $T$ would add a no-abort tuple that would prevent $A^{*}$ from aborting the transaction at any point in the future, thus allowing $B$ to obtain $s_{A}$, and hence $\sigma_{A}$, via B-resolve should it need to. The rest of the proof goes exactly the same, mutatis mutandis, as in the case above where $A$ was honest.

\section{References}

1. N. Asokan, M. Schunter, and M. Waidner. Optimistic protocols for fair exchange. In 4th ACM Conference on Computer and Communication Security, pages 6-17, 1997. 
2. N. Asokan, V. Shoup, and M. Waidner. Optimistic fair exchange of digital signatures. IBM Research Report RZ 2973, available on-line at ww.cs. visc.edu/ shoup, 1997.

3. N. Asokan, V. Shoup, and M. Waidner. Asynchronous protocols for optimistic fair exchange. In Proc. IEEE Symp. on Research in Security and Privacy, 1998. Available on-line at vww.cs.visc.edu/ ${ }^{\sim}$ shoup.

4. M. Bellare and S. Goldwasser. Encapsulated key escrow. Preprint, 1996.

5. M. Bellare and P. Rogaway. Random oracles are practical: a paradigm for designing efficient protocols. In First ACM Conference on Computer and Communications Security, 1993.

6. M. Bellare and P. Rogaway. Optimal asymmetric encryption. In Advances in Cryptology-Crypto '94, pages 92-111, 1994.

7. S. Brands. Untraceable off-line cash in wallets with observers. In Advances in Cryptology-Crypto '93, pages 302-318, 1993.

8. H. Bürk and A. Pfitzmann. Value exchange systems enabling security and unobservability. Computers and Security, 9:715-721, 1990.

9. D. Chaum and T. Pederson. Wallet databases with observers. In Advances in Cryptology-Crypto '92, pages 89-105, 1992.

10. B. Cox, J. D. Tygar, and M. Sirbu. NetBill security and transaction protocol. In First USENIX Workshop on Electronic Commerce, pages 77-88, 1995.

11. R. H. Deng, L. Gong, A. A. Lazar, and W. Wang. Practical protocols for certified electronic mail. J. of Network and Systems Management, 4(3), 1996.

12. A. Fiat and A. Shamir. How to prove yourself: practical solutions to identification and signature problems. In Advances in Cryptology-Crypto '86, pages 186-194, 1986.

13. M. K. Franklin and M. K. Reiter. Verifiable signature sharing. In Advances in Cryptology-Eurocrypt' '95, pages 50-63, 1995.

14. M. K. Franklin and M. K. Reiter. Fair exchange with a semi-trusted third party. In 4th ACM Conference on Computer and Communications Security, pages 1-5, 1997.

15. L. Guillou and J. Quisquater. A "paradoxical" identity-based signature scheme resulting from zero-knowledge. In Advances in Cryptology-Crypto '88, pages 216$231,1988$.

16. D. W. Kravitz. Digital signature algorithm, 1993. U. S. Patent No. 5,231,668.

17. C. H. Lim and P. J. Lee. More flexible exponentiation with precomputation. In Advances in Cryptology-Crypto '9/, pages 95-107, 1994.

18. S. Micali. Certified e-mail with invisible post offices. Unpublished manuscript, 1997 (presented at the 1997 RSA Security Conference).

19. H. Ong and C. Schnorr. Fast signature generation with a Fiat Shamir-like scheme. In Advances in Cryptology-Eurocrypt '90, pages 432-440, 1990.

20. C. Rackoff and D. Simon. Noninteractive zero-knowledge proof of knowledge and chosen ciphertext attack. In Advances in Cryptology-Crypto '91, pages 433-444, 1991.

21. R. L. Rivest, A. Shamir, and L. M. Adleman. A method for obtaining digital signatures and public-key cryptosystems. Communications of the ACM, pages 120-126, 1978.

22. C. Schnorr. Efficient signature generation by smart cards. J. Cryptology, 4:161$174,1991$.

23. V. Shoup. Lower bounds for discrete logarithms and related problems. In Advances in Cryptology-Eurocrypt '97, 1997. 